\title{
MyHealthAvatar and CARRE: Case Studies of Interactive Visualisation for Internet-enabled Sensor-assisted Health Monitoring and Risk Analysis
}

Article $\cdot$ September 2016

DOI: 10.1049 /iet-net.2015.0113

CITATIONS

0

11 authors, including:

\section{Enjie Liu}

University of Burdwan

35 PUBLICATIONS 98 CITATIONS

SEE PROFILE

\section{Vaidotas Marozas}

Kaunas University of Technology

76 PUBLICATIONS 229 CITATIONS

SEE PROFILE
READS

69
Arunas Lukosevicius

Kaunas University of Technology

85 PUBLICATIONS 316 CITATIONS

SEE PROFILE

\section{Eleni Kaldoudi}

Democritus University of Thrace

84 PUBLICATIONS 611 CITATIONS

SEE PROFILE

Some of the authors of this publication are also working on these related projects:

Project MyHealthAvatar View project 


\title{
MyHealthAvatar and CARRE: Case Studies of Interactive Visualisation for Internet-enabled Sensor-assisted Health Monitoring and Risk Analysis
}

\author{
Youbing Zhao ${ }^{1}$, Farzad Parvinzamir ${ }^{1}$, Zhikun Deng ${ }^{1}$, Hui Wei ${ }^{1}$, Xia Zhao ${ }^{1}$, Enjie Liu ${ }^{1}$, Feng \\ Dong $^{1 *}$, Gordon Clapworthy ${ }^{1}$, Arūnas Lukoševičius ${ }^{2}$, Vaidotas Marozas ${ }^{2}$, Eleni Kaldoudi ${ }^{3}$ \\ ${ }^{1}$ Department of Computer Science and Technology, University of Bedfordshire, Luton, UK \\ ${ }^{2}$ Biomedical Engineering Institute, Kaunas University of Technology, Kaunas, Lithuania \\ ${ }^{3}$ Physics of Medical Imaging \& Telemedicine, Schoo of Medicine, Democritus University of \\ Thrace Dragana, Alexandroupoli, Greece \\ *feng.dong@beds.ac.uk
}

\begin{abstract}
With the progress of wearable sensor technologies, more wearable health sensors have been made available on the market, which enables not only people to monitor their health and lifestyle in a continuous way but also doctors to utilise them to make better diagnoses. Continuous measurement from a variety of wearable sensors implies that a huge amount of data needs to be collected, stored, processed and presented, which cannot be achieved by traditional data processing methods. Visualisation is designed to promote knowledge discovery and utilisation via mature visual paradigms with well-designed user interactions and has become indispensable in data analysis. In this paper we introduce the role of visualisation in wearable sensor-assisted health analysis platforms by case studies of two projects funded by the European Commission: MyHealthAvatar and CARRE. The former focuses on health sensor data collection and lifestyle tracking while the latter aims to provide innovative means for the management of cardiorenal diseases with the assistance of wearable sensors. The roles of visualisation components including timeline, parallel coordinates, map, node-link diagrams, Sankey diagrams, etc. are introduced and discussed.
\end{abstract}

\section{Introduction}

The internet presents opportunities and challenges for better healthcare in the information age [1]. Traditionally most health data are acquired by dedicated medical devices during infrequent or emergent visits to clinics and hospitals in medical practice. With the increasing popularity of wearable sensors and mobile apps to capture life-logging personal health data $[2,3,4]$, people nowadays collect more life-style and health data from wearable devices and mobile apps, such as Fitbit [5], Withings [6], iHealth [7], Moves [8], etc., which provide continuous monitoring of people's long-term lifestyle and health status. This enables not only people to monitor their health in a continuous way but also provides clinicians the potential to utilise them to assist diagnosis and decision-making.

Though today's wearable sensors can only measure simple fitness data such as steps, weight, sleep quality or some basic health data such as blood pressure, heart rate, etc., there is a vision that, with the development of wearable sensor technologies, there will be a convergence of health sensors and medical sensors. Data that can only been measured and monitored with dedicated medical devices at hospitals at 
present might be accessible from portable sensors or embedded chips in the future, which may bring the fusion of personal health information and medical sensor data. More data sources, more frequently measured long-term health data and more personalised data will help not only the user to monitor their health better but will also enable clinicians to achieve better risk assessment, disease prevention and more accurate diagnosis. Effective collection of these long-term health-status data will also contribute to interdisciplinary healthcare research and collaboration in supporting innovative medical care. It meets the goal of predictive, preemptive, preventive, personalised and participative healthcare.

The continuous measurements of a variety of health and medical indicators implies that a huge amount of data from a large number of sensors needs to be collected, stored, processed and presented. Visualisation provides technologies to present the data via mature visual paradigms and well-designed user interactions. Without proper design of visualisation and user interaction, it is not possible for the user to select, view, understand and gain knowledge from a large collection of health data. As pointed out in [9], "Visualization and visual analytics researchers can contribute substantial technological advances to support the reliable, effective, safe, and validated systems required for personal health, clinical healthcare, and public health policymaking."

In this paper we introduce the role of visualisation components in a wearable sensor-assisted mobile health system by case studies of two projects funded by the 7th Framework Programme of European Commission: MyHealthAvatar [10, 11, 12] and CARRE [13, 14]. MyHealthAvatar focuses on health sensor data collection and lifestyle tracking to promote people's wellbeing while the more medical-oriented CARRE aims to provide innovative means for the management of cardiorenal diseases with the assistance of wearable sensors.

In the case studies, the roles of visual analytics components including timeline, parallel coordinates, map, node-link diagrams, etc. are introduced and discussed. The remainder of the paper is organised as follows: section 2 introduces MyHealthAvatar and CARRE, followed by related background in section 3; section 4 introduces major visualisation components employed by MyHealthAvatar and CARRE, and section 5 presents the discussions and draws the conclusion.

\section{MyHealth Avatar and CARRE}

\subsection{MyHealthAvatar}

MyHealthAvatar is a European Commission funded project aimed to provide a unique interface that allows data access, collection, sharing and analysis to overcome the shortcomings of the existing, highly fragmented resources. MyHealthAvatar believes that healthcare should not only care for patients but also look after the health and wellbeing of all people. Health data needs to be available to healthy people through 
maintenance of a healthy lifestyle and the notification of early symptoms. However, it is almost impossible for the patient or doctors themselves to aggregate the large amount of fragmented data collected from different sources and make effective analyses. Hence, MyHealthAvatar targets both healthy people and patients and is designed to be the people's lifelong companion, providing long-term and consistent healthstatus information of the individual.

As a project focuses on health self-monitoring, MyHealthAvatar is more fitness and lifestyle oriented. The visualisation components in MyHealthAvatar to facilitate health and lifestyle data presentation and analysis include timeline, clock view, map, activity graph, etc., which can be integrated to achieve flexible visualisation of spatio-temporal lifestyle data.

MyHealthAvatar provides health data collection, storage and access to people, patients and doctors. The data can either be automatically collected or manually input. In MyHealthAvatar, there are several model-based tools to help users assess the risks of cardiovascular disease, hypertension, diabetes, and stroke. For example, based on the Framingham model [15] and the data collected from sensors, diabetes risk can be evaluated dynamically.

\section{2. $C A R R E$}

Different from MyHealthAvatar, CARRE aims to provide personalized empowerment and shared decision support services for cardiorenal disease comorbidities based on risk models extracted from medical publications. The chronic cardiorenal disease is characterized by simultaneous kidney and heart disease while the primarily failing organ may be either the heart or the kidney. In CARRE, sources of medical and other knowledge are semantically linked with sensor outputs to provide clinical information personalised to the individual patient, so as to be able to track the progression and interactions of comorbid conditions. The ultimate goal is to provide the means for patients with comorbidities to take an active role in care processes, including self-care and shared decision-making, and also to support medical professionals in understanding and treating comorbidities via an integrative approach.

The CARRE repository [16] stores general medical knowledge relating to risk associations, evidence and observables, which forms a large semantic graph structure data that are either related to ground knowledge in cardio-renal disease and comorbidities (symptoms, diseases, risk factors, treatments, medical evidence source data, educational content, etc.) or personalised to each patient (patient demographics, medical history, sensor data, lifestyle data, etc.).

The key concepts in the CARRE risk factor network are defined as follows:

Risk Element: Risk elements include all the disorders/diseases involved in the comorbidity under discussion as well as any other risk causing agent, e.g. demographic (e.g. age, sex, race), genetic (gene 
polymorphisms), behavioural (e.g. smoking, physical exercise), environmental (e.g. air pollution, allergens) or even an intervention (e.g. pharmaceutical substances, contrast agents).

Risk Association: The association of one risk element as the risk source with another risk element, which is the negative outcome under certain conditions, is a 'risk association'. A source risk element can be associated with a target risk element with more than one risk association.

Risk Observable: In a risk association the prerequisite circumstances relate directly to the existence of the risk agent (source risk target) and/or its severity, and/or any other specific conditions. These are reported via certain 'observables', that is, physical variables that can be measured or otherwise ascertained (e.g. biomarkers, biometric variables, biological signals and other non-biological factors, e.g. environmental).

Currently there are 98 risk factors, 53 risk elements, 253 risk evidences, 63 observables and 60 evidence sources in the CARRE risk data repository.

Visualisation is employed so that patients and clinicians will be able to view, understand and interact with this linked knowledge and also take advantage of personalised empowerment services supported by a dedicated decision support system. The aim is to help medical professionals better understand the individual patient's disease development and help patients to understand their own disease development, which in turn assists them to conform to the self-management plan.

\section{Related Background}

3.1. Wearable sensors, mobile apps and web platforms for health data collection

Wearable device based health data collection tools such as Fitbit, Withings and iHealth sensors record steps, distance, calories, heart rate, sleep quality, blood pressure, and weight. Moves and the Google Map timeline [17] record the user's location and daily routes, and can recognise some of the user's activities. The collected data can be uploaded to the system's servers and can be shared via APIs with other internet applications.

Currently MyHealthAvatar supports importing data directly from Fitbit, Withings and Moves. CARRE, by connecting to Microsoft HealthVault [18], supports more devices and apps in addition to the data sources it directly accesses, including Fibit, Withings and iHealth, etc.

Fitbit provides wearable devices which record steps, distance, and calories, etc. These devices communicate with a host computer using Bluetooth that sends their data directly to a user's account on the Fitbit website.

Withings provides devices that measure or calculate steps, distance, calories, heart rate, sleep quality, heart rate etc. Devices include wristband, watch, scales, blood pressure monitor. 
Moves is an app for fitness and lifestyle recording. It automatically records the step number and location of the user and automatically recognises the activity type, such as walking, running, cycling, transport, etc. The user can either view the distance, duration, steps, and calories data on the mobile phone or export the data from the Moves server. An automatic daily storyline with time and location are recorded and shown on the map in the Moves app.

On the other hand, there has been increasing interest in building centralised health and fitness data repositories. HealthVault integrated a number of fitness sensors on the market to provide a central repository and APIs for data access, but its emphasis is on data storage and does not provide powerful interactive visualisation tools for data analysis. Fluxtream [19] is an open source project to provide data storage of fitness sensors and basic visualisation tools.

\subsection{Visualisation for healthcare}

Health data, especially electronic health records (EHRs), as an important type of time-oriented data, has become the topic of visualisation research for several decades [20]. Rind et. al. [21] has presented a detailed review of EHR visualization techniques. West et al. [22] also present a systematic review of innovative visual analytics approaches that have been proposed to illustrate EHR data.

Lifelines [23] is a pioneer work in visualisation of individual patient records, which provides a general visualisation environment for problems, diagnoses, test results or medications using timelines. Lifelines2 [24] provides visualisation of temporal categorical data across multiple records, which is better for a doctor to view to discover and explore patterns across these records to support hypothesis generation, and find cause-and-effect relationships in a population. LifeFlow [25] and EventFlow [26] are tools for event sequence analytics for a group of patients. They extract and highlight the common event sequence from patient records.

Outflow [27] and DecisionFlow [28] uses Sankey diagram [29] style visualization to help visualise and analyse the causal relationships of events in complex event sequences. VISITORS [30], which is based on KNAVE [31] and KNAVE II [32], uses aggregation to extract meaningful interpretations from multiple patients' raw time-oriented data. PatternFinder [33] provides tools for the user to query patterns by specifying the attributes of events and time spans.

\section{Visualisation Components}

Visualisation uses effective visual encoding and user interaction to represent data in a visually understandable means. It is designed to promote data presentation and knowledge discovery via effective visual paradigms and well-designed user interactions. In this section, the main visualisation components employed by MyHealthAvatar and CARRE are introduced and discussed. 


\subsection{Timeline for sensor measurement visualisation}

Most of the health data and sensor data are time-dependent. A timeline is a traditional method to visualise time-varying data and events in a linear layout which is suitable for continuous variables which may cover a relatively long period, such as health indicators and medical measurements. Thus, timeline is employed by both MyHealthAvatar and CARRE.

The application of the timeline is mostly for visualisation and analysis of the fitness statistics and the medical indicators. Data trends can be observed from the variable curves and data correlations may be discovered by comparison of the data curves of the multi-variables. As the data records may cover a long time range, interactive techniques such as zooming and overview+details [34] are integrated with the visualisation. Bar charts and lines are available for variable visualisation. As there may be a number of variables to select, a convenient drag-and-drop is used for variable selection. Fig. 1 shows multiple health indicators visualised in an interactive timeline in CARRE.

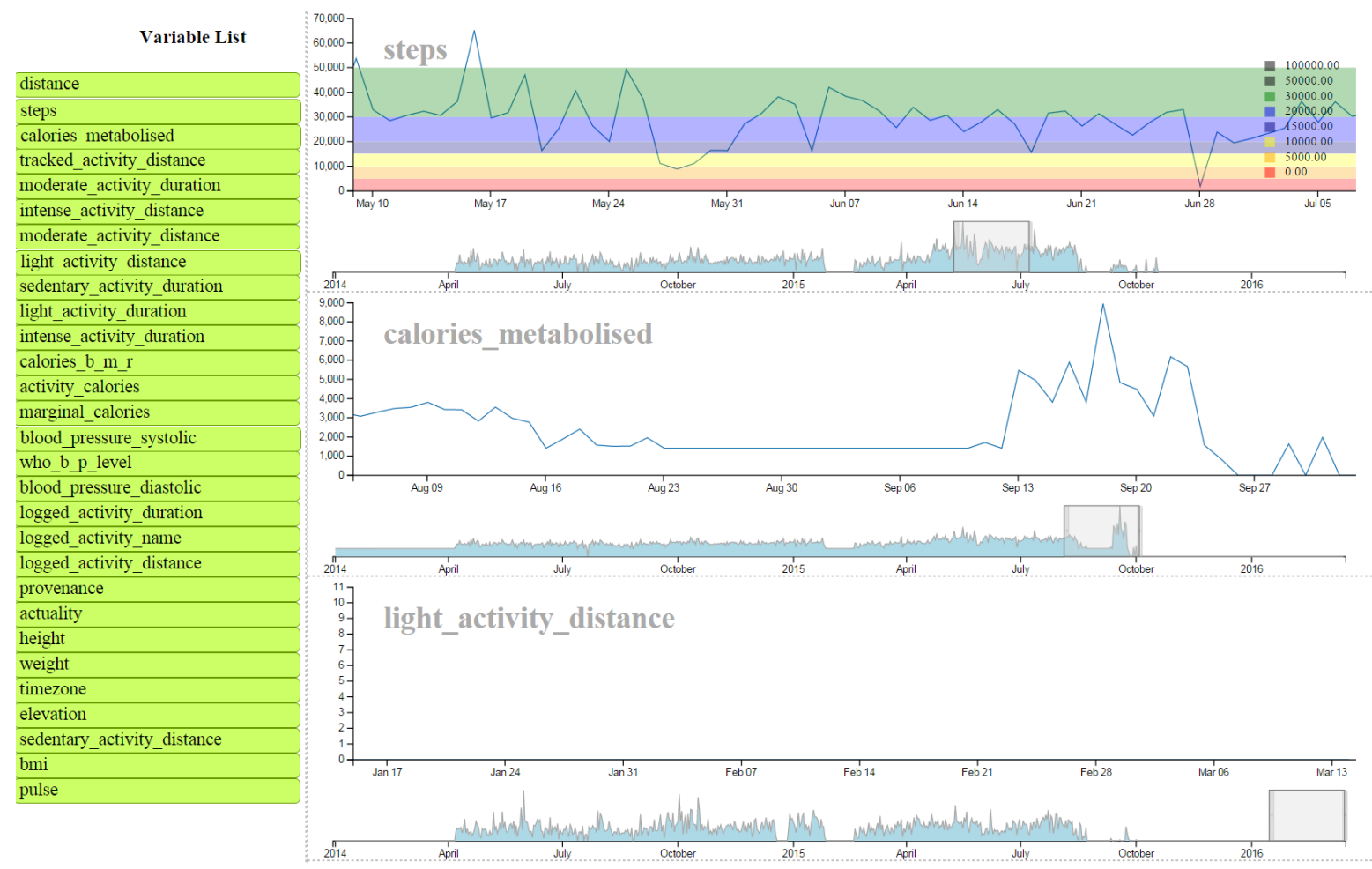

Fig. 1. A timeline view of health indicators

\subsection{Visualisation of Time-dependant Data in a Radial Layout}

For daily activities, timeline provides linear visualisation over a relatively long period. Interactive timelines provide zooming to smaller scales. However, the linear layout may make it difficult for the user to understand and compare periodic events. A fine-grained view of activities within one day is better visualised in a radial layout. A real-life way of radial daily time representation is the clock. MyHealthAvatar 
employs a similar radial layout called clock view to visualise daily events. Activity types are marked by icons and colours. When the user hovers the mouse over icons and arcs more detailed information will be displayed, as shown in Fig. 2.

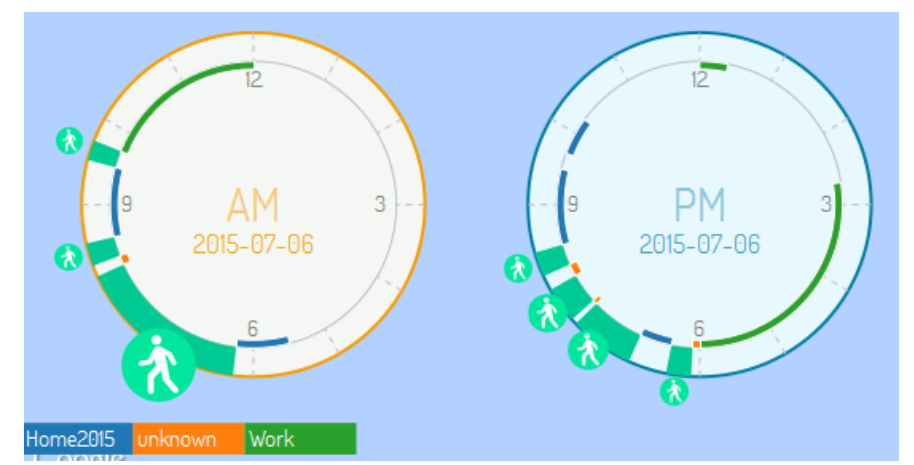

Fig. 2. Clock view of daily events in MyHealthAvatar

\subsection{Map for location and movement visualisation}

While the timeline and clock view are mainly designed for visualisation of temporal data, they are not suitable for visualization of spatial locations and events. A map is a natural choice to provide intuitive spatiotemporal visualisation and analysis of the user's locations and routes for better understanding and knowledge discovery of the lifestyle. In MyHealthAvatar the map is used for visualisation and analysis of the Moves places and tracks, but it is capable of supporting other location-sensor-based apps. The current map implementation is based on Google Maps [35]. Doughnut chart based clustering is provided for place visualisation and transparent routes are used for visualisation of multiple day routes, as shown in Fig. 3.

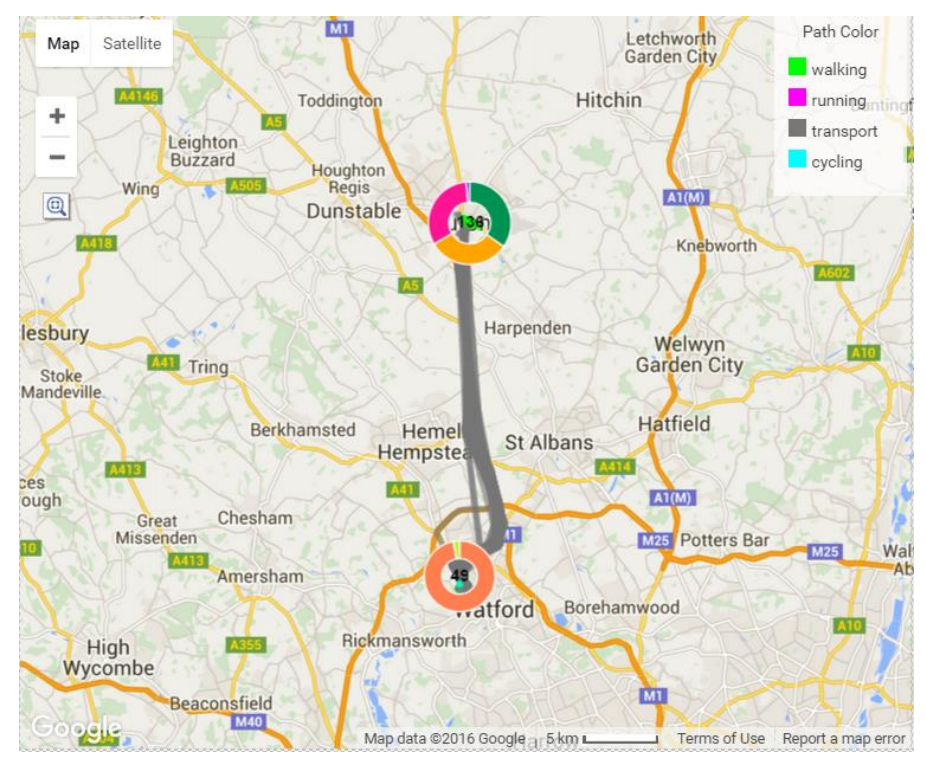

Fig. 3. Map visualisation of Moves tracking routes in MyHealthAvatar

\subsection{Parallel coordinates for multivariate analysis}


The technique of parallel coordinates is an approach for visualising multiple quantitative variables using multiple axis which are placed parallel to each other in the most common case [36]. The advantage of parallel coordinates is that it supports visualisation of multiple variables and correlation between attributes can be discovered by certain visualisation patterns. It is a common technique of visualising high-dimensional data and analysing multivariate data.

Currently parallel coordinates is employed by CARRE for multi-variable correlation analysis of the biomarkers. An example view of the parallel coordinate view is shown in Fig. 4 where negative correlations can be found between walking minutes and blood pressures as well as BMI (Body Mass Index).

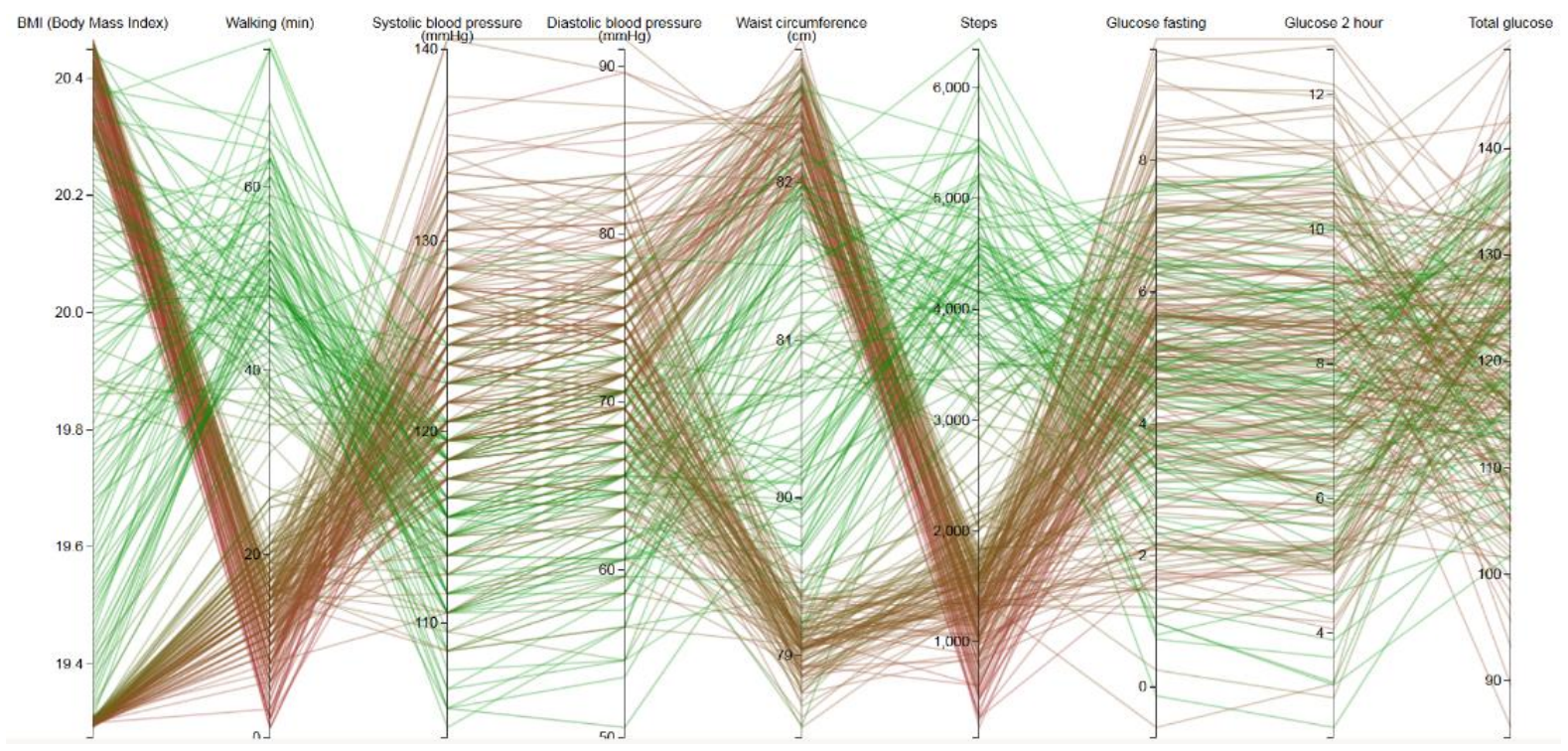

Fig. 4. Health indicator correlation analysis based on Parallel Coordinates in CARRE

\subsection{Node-link Diagram}

The node-link diagram [37] is a traditional technique to visualise relationships visually where point markers represent nodes in the graph and line segments connecting nodes represent edges in the graph. Node-link diagrams can be used to visualise trees and general network data structure. Different layout algorithms can be applied to minimize the number of distracting artefacts such as edge crossings. Forcedirected layout algorithms [38] have been proved to be flexible and effective for dynamic and interactive graphs.

Fig. 5 shows a force-directed node-link diagram visualisation of personalised risk elements and risk factor connections in CARRE repository. Each node represents a risk element and each link represents a risk factor. By viewing the graph, a normal patient can easily know his major risks in a more intuitive manner. 


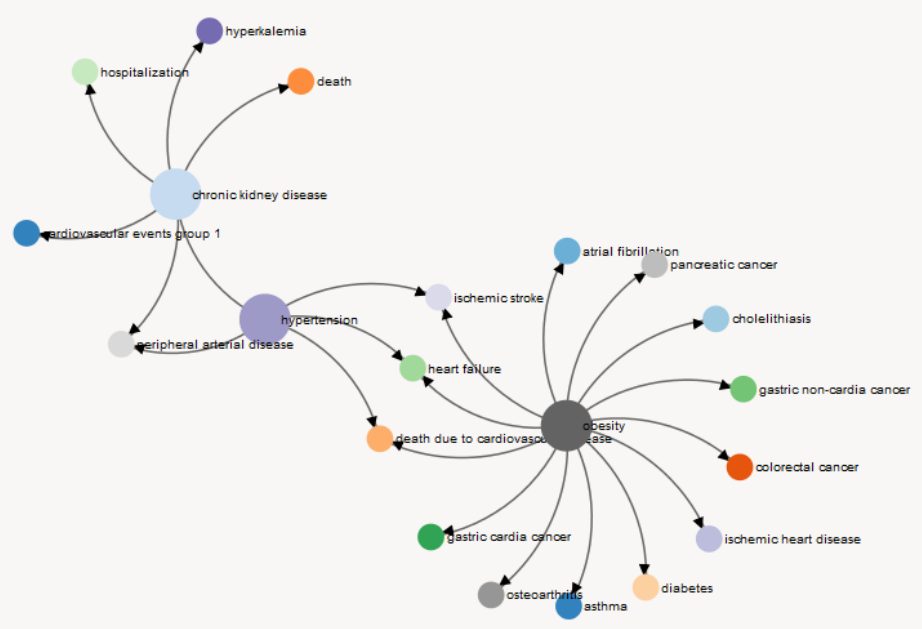

Fig. 5. Force layout based Node-link diagram of risk factors filtered according to the conditions of a particular patient in CARRE

A node-link diagram is also used for visual analysis of frequent routes and places in MyHealthAvatar. An example visualisation is shown in Fig. 6 where the nodes represent places and links represent the routes. The colour of the nodes represents different place types and the link width and transparency represent the route frequency. From the visualisation frequent routes can be easily recognised.

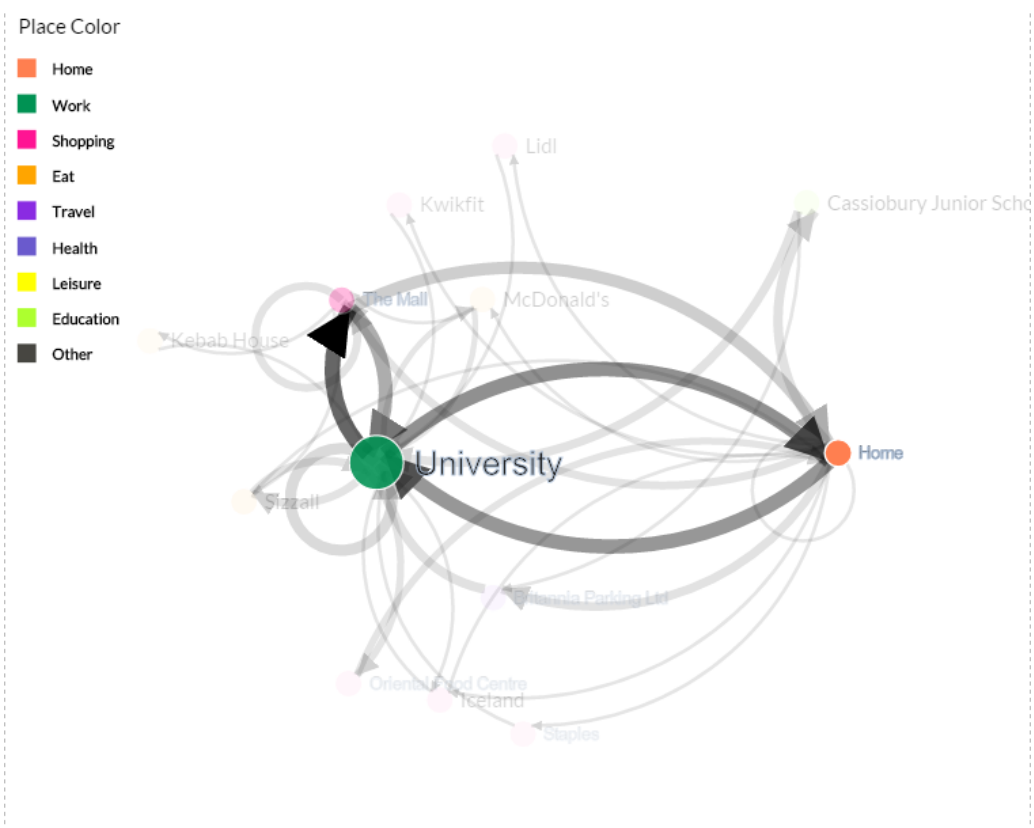

Fig. 6 Visualisation of frequent routes and places in MyHealthAvatar 


\subsection{Chord diagram}

The disadvantage of the node-link diagram is that without proper handling, as the number of nodes and links increase, the visualisation will become increasingly messy for effective viewing. Though filtering can be applied based on conditions of a particular patient, it does not help for the visual analysis of the whole risk factor database. Fortunately there are some network visualisation techniques to alleviate the problem, such as the chord diagram [39], as shown in Fig. 7. In the chord diagram, the risk elements are placed on the circle and the chords represent the risk factors. Relationships of all risk elements can be clearly presented. The risk associations with the specified risk element are highlighted when the user hovers the mouse on the element. The chord diagram is particularly useful for professionals to check and insert new risks.

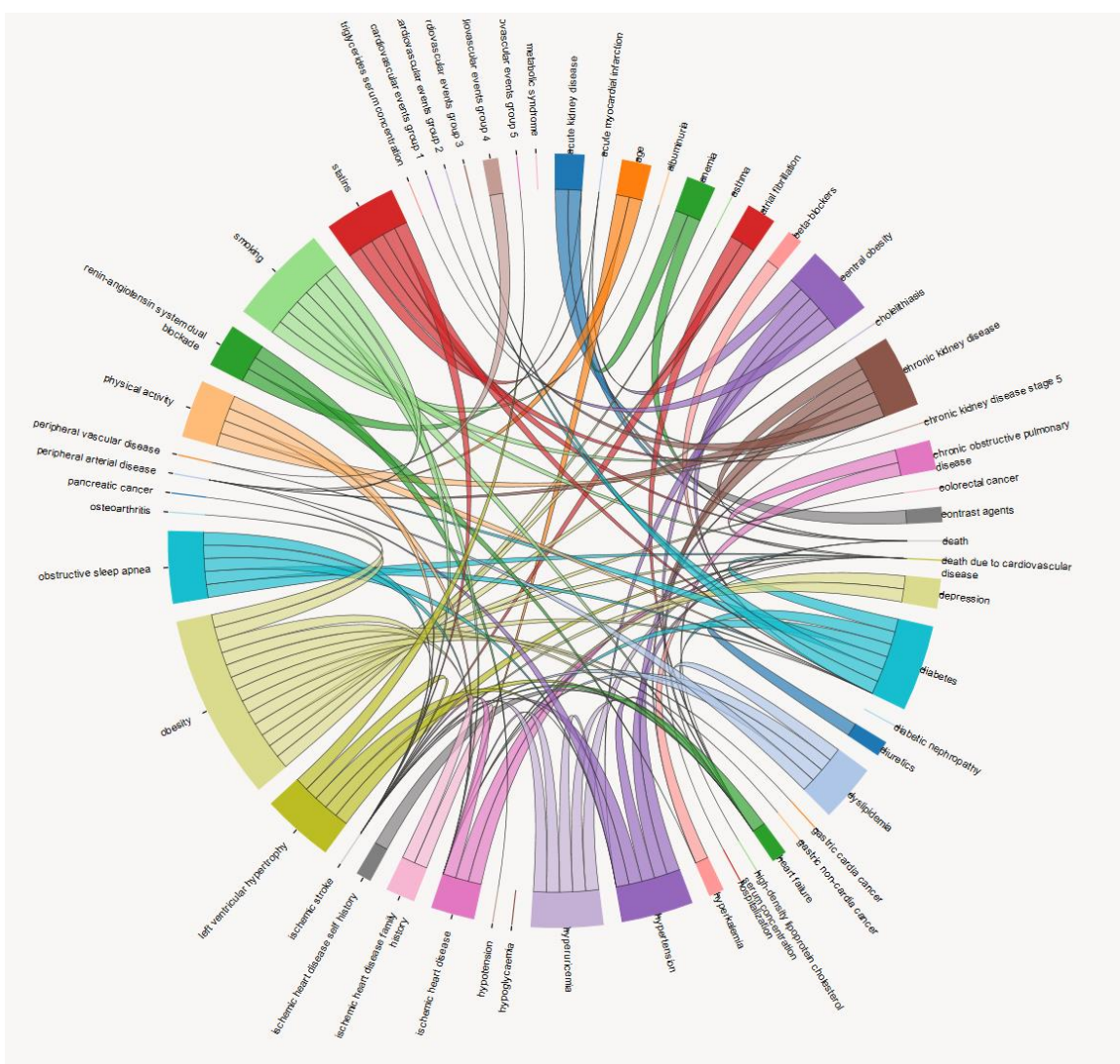

Fig. 7. Chord diagram of risk elements and risk factors in CARRE

\subsection{Sankey diagram}

The disadvantage of the chord diagram is that it can only show the associations between the risk elements but cannot show the time order of risk propagations in a static way. A Sankey diagram is a better choice for visualising causal relationships. As introduced in the related work, OutFlow and DecisionFlow use Sankey diagram style visualisation to visually analyse the causal relationships of events. The advantage of the Sankey diagram is that it shows the multi-layer causal relationships of the elements in a much more 
clear and understandable way than the node-link diagram, though it is not suitable for visualisation of general graph data. Fig. 8 shows the Sankey diagram for risk visualisation and analysis in CARRE. The leftmost nodes represent the risk observables and other nodes are risk elements. It is fairly easy to identify the major risk observables relating to a disease (risk element) and to recognise the routes of risk propagation from the visualisation.

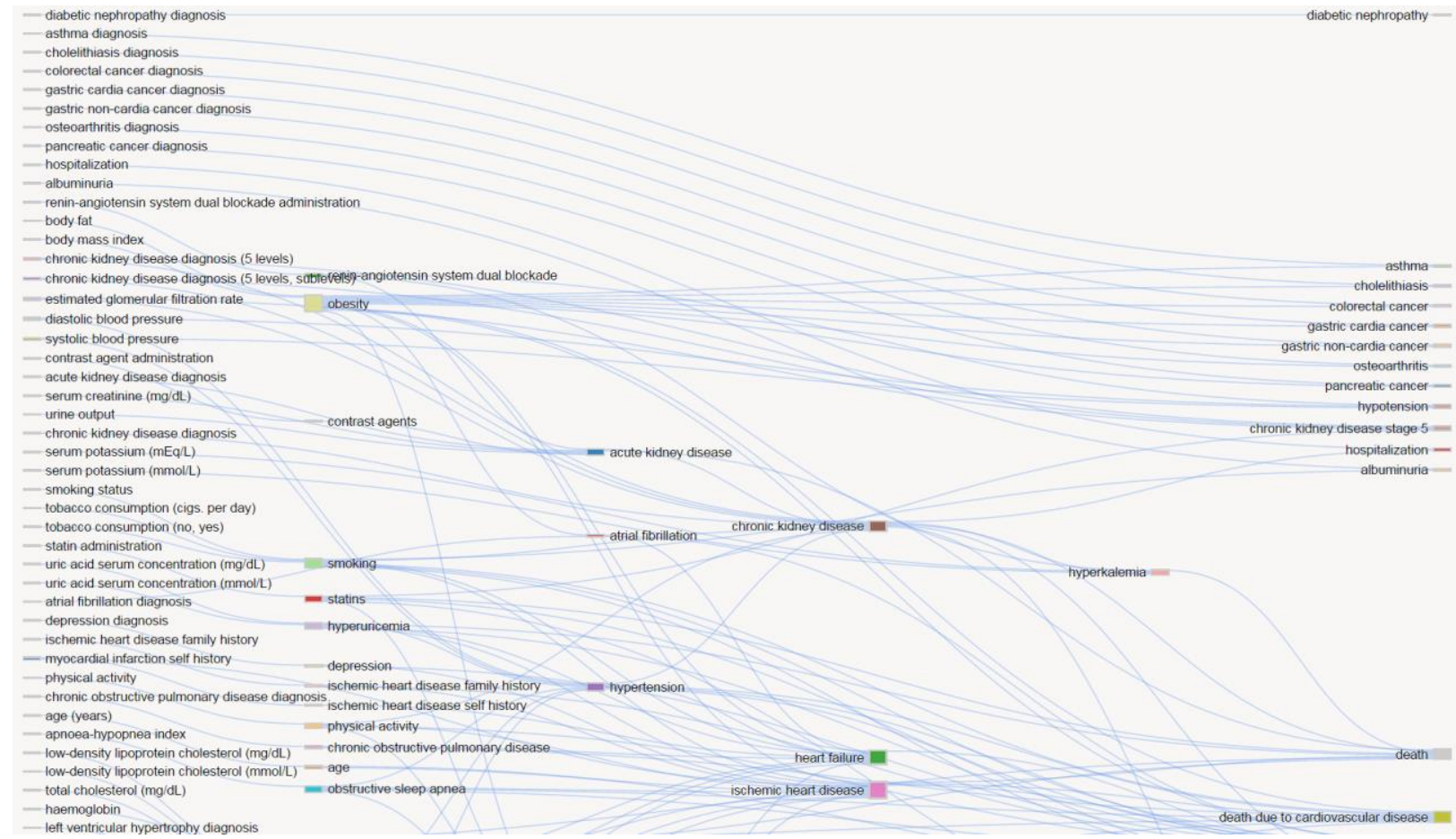

Fig. 8. Sankey diagram for risk analysis in CARRE

\section{Discussions and Conclusions}

With the increasing popularity of wearable sensors, people are more interested in studying the applications of the sensors for lifestyle tracking and decision support for professional clinicians. While the data amount collected by wearable sensors is huge, without proper visualisation it is almost not possible to carry out any effective data analysis.

The two projects MyHealthAvatar and CARRE have differences and commonalities indicated here.

\section{Differences:}

MyHealthAvatar is more lifestyle analysis oriented. Consequently it needs to collect and visualise the place and movement tracking, while in CARRE no map visualisation is needed. Maps are intuitive for visualisation of daily events and routes in MyHealthAvatar.

The central data repository of CARRE is the risk factor database which contains relationships of different risk elements. Thus, CARRE is highly dependent on relationship visualisation and analysis. In CARRE, node-link diagram, chord diagram, Sankey diagram are used while in MyHealthAvatar only the 
node-link diagram is used for visualisation of frequent routes and places. Chord diagram and Sankey diagram are more preferable over node-link diagram when the number of nodes becomes large. Also, Sankey diagram is especially useful for causal analysis and risk propagation analysis.

\section{Commonalities:}

Both MyHealthAvatar and CARRE are backed by health sensor data. Thus, visualisation of timeoriented data collected from health sensors is needed. Time-dependant visualisation in a linear timeline or a radial layout helps to view and analyse events and health indicators in both MyHealthAvatar and CARRE, especially when proper user interaction techniques are incorporated.

Both MyHealthAvatar and CARRE use certain models to support risk analysis. In MyHealthAvatar the Flamingham model is used and in CARRE a graph based model extracted from medical literatures is used.

Parallel coordinate is very useful for correlation analysis of multiple attributes in both projects, though currently it is only employed in CARRE.

In conclusion, interactive visualisation of time-oriented sensor data, location aware activity data and risk model data not only helps data presentation for convenient data understanding but also promotes risk analysis in health sensor backed health monitoring and risk evaluation platforms.

\section{Acknowledgments}

MyHealthAvatar project is funded by 7th Framework Programme of European Commission - ICT under agreement of FP7-ICT-2011-9. CARRE project is funded by 7th Framework Programme of European Commission - ICT under agreement of FP7-ICT-611140. We would like to thank the European Commission for the funding and thank the project officers and reviewers for their indispensable support for both of the projects.

\section{References}

[1] Peter Groves, Basel Kayylai, David Knott and Steve Van Kuiken, The 'big data' revolution in healthcare (McKinsey \& Company, 2013) url: http://www.mckinsey.com

[2] Qi, J., Yang, P., Fan, D. Fan, Deng, Z., "A survey of Physical Activity Monitoring and Assessment Using Internet of Things technology", the 2015 IEEE International Conference on Pervasive Intelligence and Computing, Liverpool, UK, pp. 2353-2358, October, 2015.

[3] Deng, Z., Yang, P., Zhao, Y., Zhao, X., Dong, F., "Life-logging data aggregation solution for interdisciplinary healthcare research and collaboration", the 2015 IEEE International Conference on Pervasive Intelligence and Computing, pp. 2315-2320, Liverpool, UK, October, 2015.

[4] Yang, P., Hanneghan M., Qi J., Deng Z., Fan D., Dong F., "Improving the Validity of Lifelogging Physical Activity Measures in an Internet of Things Environment", pp. 2309-2314, the 2015 IEEE International Conference on Pervasive Intelligence and Computing, Liverpool, UK, October, 2015.

[5] Fitbit, url: http://www.fitbit.com/

[6] Withings, url: http://www.withings.com/

[7] iHealth, url: http://www.ihealthlabs.com/

[8] Moves url: https://www.moves-app.com/

[9] Shneiderman, B., Plaisant, C., Hesse, B.W.: 'Improving health and healthcare with interactive visualization methods', IEEE Computer, 2013, 46, (1), pp. 58-66 
[10] 'MyHealthAvatar Project', url: http://www.myhealthavatar.eu/

[11] 'MyHealthAvatar Platform', url: http://www.myhealthavatar.org/

[12] Spanakis E. G., Kafetzopoulos D., Yang P., Marias K., Deng Z., Tsiknakis M., Sakkalis V.,Dong F., "MyHealthAvatar: Personalised and empowerment health services through Internet of Things technologies", the 2014 EAI 4th International Conference on Wireless Mobile Communication and Healthcare, Athens, Greece, November, 2014.

[13] 'CARRE project', url: http://www.carre-project.eu/

[14] 'CARRE Visual Analytics', url: http://visual.carre-project.eu/

[15] Wilson, P.F., Meigs, J.B., Sullivan, L. et al.: 'Prediction of Incident Diabetes Mellitus in Middle-aged Adults: The Framingham Offspring Study', Arch Intern Med., 2007, 167, (10), pp. 1068-1074

[16] Kaldoudi, E., Third, A., Gotsis, G. et al.: CARRE Deliverable D.2.2: Functional Requirements \& CARRE information model (July 2015) url: https://www.carre-project.eu/project-info/deliverables/, CARRE: Personalized Patient Empowerment and Shared Decision Support for Cardiorenal Disease and Comorbidities, FP7-ICT-61140

[17] Google Maps Timeline, url: https://www.google.com/maps/timeline

[18] HealthVault url: https://www.healthvault.com/

[19] Fluxtream url: https://fluxtream.org/

[20] Aigner, W., Miksch, S., Schumann, H. et al.: Visualization of Time-Oriented Data. Human-Computer Interaction Series (Springer, 2011)

[21] Rind, A., Wang, T.D., Shneiderman, B.: nteractive information visualization to explore and query electronic health records', Foundations and Trends in HCI, 2011, 5, (3), pp. 207-298

[22] West, V.L., Borland, D., Hammond, W.E.: 'Innovative information visualization of electronic health record data: a systematic review', Journal of the American Medical Informatics Association, Mar 2015, 22, (2)

[23] Plaisant, C., Mushlin, R., Snyder, A.et al.: 'Lifelines: Using visualization to enhance navigation and analysis of patient records'. In Proc 1998 American Medical Informatic Association Annual Fall Symposium, 1998, pp. 76-80

[24] Wang, T.D., Plaisant, C., Shneiderman, B. et al.: 'Temporal summaries: Supporting temporal categorical searching, aggregation and comparison', IEEE Transactions on Visualization and Computer Graphics, Nov 2009, 15, (6), pp. 1049-1056

[25] Wongsuphasawat, K., Guerra Gómez, J.A., Plaisant, C. et al.: 'Lifeflow: Visualizing an overview of event sequences'. Proc SIGCHI Conf.on Human Factors in Comp. Sys., New York, NY, 2011, pp. 1747-1756

[26] Monroe, M., Lan, R., Lee, H. et al.: 'Temporal event sequence simplification', JOURNAL, 2013, Vol (2)

[27] Wongsuphasawat, K., Gotz, D.: 'Exploring flow, factors, and outcomes of temporal event sequences with the outflow visualization', IEEE Trans. on Visualization and Comp. Graphics, Dec 2012, 18, (12), pp. 2659-2668

[28] Gotz, D.: Stavropoulos H.: Decisionflow: 'Visual analytics for high-dimensional temporal event sequence data', IEEE Trans. on Visualization and Comp. Graphics, 2014, 20 (12)

[29] Riehmann, P., Hanfler, M., Froehlich, B.: 'Interactive Sankey diagrams'. Proc. IEEE Symp. on Info. Visualization, , location, month 2005, pp. 233-240

[30] Klimov, D., Shahar, Y., Taieb-Maimon, M.: 'Intelligent visualization and exploration of time-oriented data of multiple patients', Artificial Intelligence in Medicine, 2010, 49, (1), pp. 11-31

[31] Shahar, Y., Cheng, C.: 'Intelligent visualization and exploration of time-oriented clinical data'. Proc. 32nd Annual Hawaii International Conf. on Systems Sciences, Hawaii, Jan 1999

[32] Shahar, Y., Goren-Bar, D., Boaz, D. et al.: 'Distributed, intelligent, interactive visualization and exploration of time-oriented clinical data and their abstractions', Artificial Intelligence in Medicine, 2006, 38, (2), pp. 115-135

[33] Fails, J.A., Karlson, A.K., Shahamat, L. et al.: 'A visual interface for multivariate temporal data: Finding patterns of events across multiple histories', in Wong, P.C., Keim, D.A. (Eds.) 'IEEE VAST' (IEEE Computer Society, 2006) pp. 167-174

[34] Cockburn, A., Karlson, A., Bederson, B.B.: 'A review of overview+detail, zooming, and focus+context interfaces', ACM Computing Surveys (CSUR) Surveys, 2009, 41, (1)

[35] Google Maps url: https://maps.google.com

[36] Inselberg, A., Dimsdale, B.: 'Parallel coordinates: A tool for visualizing multi-dimensional geometry'. Proc. $1^{\text {st }}$ IEEE Symp. on Visualization, Oct. 1990, pp. 361-378

[37] Liu, S., Cui, W., Wu, Y. et al.: 'A survey on information visualization: recent advances and challenges', The Visual Computer, 2014, 30, (12), pp. 1373-1393

[38] Fruchterman, T., Reingold, E.: 'Graph Drawing by Force-Directed Placement', Software - Practice \& Experience, 1991, 21, (11), pp. $1129-1164$

[39] Holten, D.: 'Hierarchical Edge Bundles: Visualization of Adjacency Relations in Hierarchical Data', IEEE Trans on Visualization and Computer, 2006, 12, (5) 